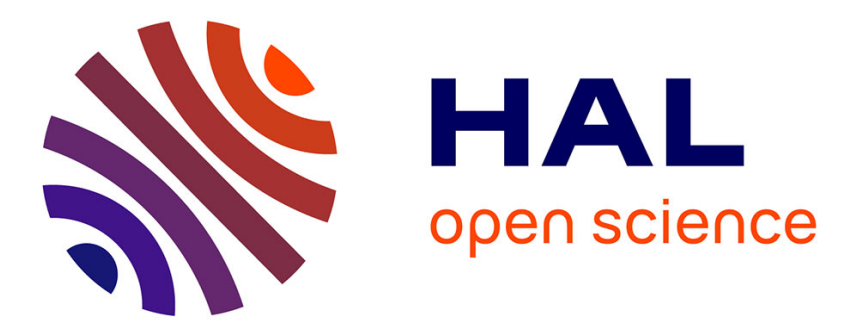

\title{
Interpolation by polynomial functions of distributive lattices: a generalization of a theorem of R. L. Goodstein
}

\author{
Miguel Couceiro, Tamas Waldhauser
}

\section{To cite this version:}

Miguel Couceiro, Tamas Waldhauser. Interpolation by polynomial functions of distributive lattices: a generalization of a theorem of R. L. Goodstein. Algebra Universalis, 2013, 69 (3), pp.13. 10.1007/s00012-013-0231-6 . hal-01090572

\section{HAL Id: hal-01090572 \\ https://hal.science/hal-01090572}

Submitted on 18 Feb 2017

HAL is a multi-disciplinary open access archive for the deposit and dissemination of scientific research documents, whether they are published or not. The documents may come from teaching and research institutions in France or abroad, or from public or private research centers.
L'archive ouverte pluridisciplinaire HAL, est destinée au dépôt et à la diffusion de documents scientifiques de niveau recherche, publiés ou non, émanant des établissements d'enseignement et de recherche français ou étrangers, des laboratoires publics ou privés. 


\title{
INTERPOLATION BY POLYNOMIAL FUNCTIONS OF DISTRIBUTIVE LATTICES: A GENERALIZATION OF A THEOREM OF R. L. GOODSTEIN
}

\author{
MIGUEL COUCEIRO AND TAMÁS WALDHAUSER
}

\begin{abstract}
We consider the problem of interpolating functions partially defined over a distributive lattice, by means of lattice polynomial functions. Goodstein's theorem solves a particular instance of this interpolation problem on a distributive lattice $L$ with least and greatest elements 0 and 1, resp.: Given a function $f:\{0,1\}^{n} \rightarrow L$, there exists a lattice polynomial function $p: L^{n} \rightarrow L$ such that $\left.p\right|_{\{0,1\}^{n}}=f$ if and only if $f$ is monotone; in this case, the interpolating polynomial $p$ is unique.

We extend Goodstein's theorem to a wider class of partial functions $f: D \rightarrow$ $L$ over a distributive lattice $L$, not necessarily bounded, and where $D \subseteq L^{n}$ is allowed to range over $n$-dimensional rectangular boxes $D=\left\{a_{1}, b_{1}\right\} \times \cdots \times$ $\left\{a_{n}, b_{n}\right\}$ with $a_{i}, b_{i} \in L$ and $a_{i}<b_{i}$, and determine the class of such partial functions which can be interpolated by lattice polynomial functions. In this wider setting, interpolating polynomials are not necessarily unique; we provide explicit descriptions of all possible lattice polynomial functions which interpolate these partial functions, when such an interpolation is available.
\end{abstract}

\section{INTRODUCTION}

Let $L$ be a distributive lattice and let $f: D \rightarrow L\left(D \subseteq L^{n}\right)$ be an $n$-ary partial function on $L$. In this paper we are interested in the problem of extending such partial functions to the whole domain $L^{n}$ by means of lattice polynomial functions, i.e., functions that can be represented as compositions of the lattice operations $\wedge$ and $\vee$ and constants. More precisely, we aim at determining necessary and sufficient conditions on the partial function $f$ that guarantee the existence of a lattice polynomial function $p: L^{n} \rightarrow L$ which interpolates $f$, that is, $\left.p\right|_{D}=f$.

An instance of this problem was considered by Goodstein $[8]$ in the case when $L$ is a bounded distributive lattice, and the functions to be interpolated were of the form $f:\{0,1\}^{n} \rightarrow L$. Goodstein showed that such a function $f$ can be interpolated by lattice polynomial functions if and only if it is monotone. Furthermore, if such an interpolating polynomial function exists, then it is unique.

The general solution to the above mentioned interpolation problem eludes us. However, we are able to generalize Goodstein's result by allowing $L$ to be an arbitrary (possibly unbounded) distributive lattice and considering functions $f: D \rightarrow L$, where $D=\left\{a_{1}, b_{1}\right\} \times \cdots \times\left\{a_{n}, b_{n}\right\}$ with $a_{i}, b_{i} \in L$ and $a_{i}<b_{i}$. More precisely, we furnish necessary and sufficient conditions for the existence of an interpolating polynomial function. As it will become clear, in this more general setting, uniqueness is not guaranteed, and thus we determine all possible interpolating polynomial functions.

2010 Mathematics Subject Classification. Primary: 06D99; Secondary: 08A40, 06E99, 28B15, 90B50.

Key words and phrases. distributive lattice, polynomial function, interpolation, disjunctive normal form, Sugeno integral.

The first named author is supported by the internal research project F1R-MTH-PUL-12RDO2 of the University of Luxembourg. The second named author acknowledges that the present project is supported by the TÁMOP-4.2.1/B-09/1/KONV-2010-0005 program of the National Development Agency of Hungary, by the Hungarian National Foundation for Scientific Research under grants no. K77409 and K83219, by the National Research Fund of Luxembourg, and cofunded under the Marie Curie Actions of the European Commission (FP7-COFUND). 
The structure of the paper is as follows. In Section 2 we recall basic background on polynomial functions over distributive lattices (see [7, 10]) and formalize the interpolation problem that we are interested in. In Section 3 we state and prove the characterization of those functions that can be interpolated by polynomial functions and we describe the set of all solutions of the interpolation problem. We discuss variations of the interpolation problem in Section 4 and relate our work to earlier results obtained for finite chains in [13. Finally, in Section 5 we consider potential applications of our results in mathematical modeling of decision making.

\section{Preliminaries}

Let $L$ be a bounded distributive lattice with least element 0 and greatest element 1. It can be shown that a function $p: L^{n} \rightarrow L$ is a lattice polynomial function if and only if there exist $c_{I} \in L(I \subseteq[n]:=\{1, \ldots, n\})$ such that $p$ can be represented in disjunctive normal form (DNF for short) by

$$
p(\mathbf{x})=\bigvee_{I \subseteq[n]}\left(c_{I} \wedge \bigwedge_{i \in I} x_{i}\right), \text { for all } \mathbf{x}=\left(x_{1}, \ldots, x_{n}\right) \in L^{n} .
$$

It is easy to verify that taking $c_{I}^{\prime}=\bigvee_{J \subseteq I} c_{J}$, we also have

$$
p(\mathbf{x})=\bigvee_{I \subseteq[n]}\left(c_{I}^{\prime} \wedge \bigwedge_{i \in I} x_{i}\right)
$$

and hence the coefficients $c_{I}$ can be assumed to be monotone in the sense that $I \subseteq J$ implies $c_{I} \leq c_{J}$. This monotonicity assumption allows us to recover the coefficients of the DNF from certain values of the polynomial function $p$. Indeed, denoting by $\mathbf{1}_{I}$ the characteristic vector of $I \subseteq[n]$ (i.e., the tuple $\mathbf{1}_{I} \in L^{n}$ whose $i$-th component is 1 if $i \in I$ and 0 if $i \notin I)$, we then have that $p\left(\mathbf{1}_{I}\right)=c_{I}$. Thus each polynomial function $p$ has a unique DNF with monotone coefficients. We call such a DNF a maximal disjunctive normal form, as the coefficients of this DNF are the greatest among all DNF's representing the same polynomial function $p$ (see [1]). In the sequel we will always consider lattice polynomial functions in maximal DNF. These observations contain the essence of Goodstein's theorem. Let us note that this result is implicit in the proof of [9, Corollary 2].

Theorem 2.1 (Goodstein [8]). Let $L$ be a bounded distributive lattice, and let $f$ be a function $f:\{0,1\}^{n} \rightarrow L$. There exists a polynomial function $p$ over $L$ such that $\left.p\right|_{\{0,1\}^{n}}=f$ if and only if $f$ is monotone. In this case $p$ is uniquely determined, and can be represented by the maximal DNF

$$
p(\mathbf{x})=\bigvee_{I \subseteq[n]}\left(f\left(\mathbf{1}_{I}\right) \wedge \bigwedge_{i \in I} x_{i}\right)
$$

Remark 2.2. The notion dual to disjunctive normal form is that of conjunctive normal form. Every polynomial function over a bounded distributive lattice has a unique minimal conjunctive normal form whose coefficients satisfy $I \subseteq J \Longrightarrow c_{I} \geq$ $c_{J}$. The minimal conjunctive normal form of the polynomial function $p$ in Theorem 2.1 is

$$
p(\mathbf{x})=\bigwedge_{I \subseteq[n]}\left(f\left(\mathbf{1}_{I}\right) \vee \bigvee_{i \notin I} x_{i}\right) .
$$

Informally, Goodstein's theorem asserts that polynomial functions of distributive lattices are uniquely determined by their restrictions to the hypercube $\{0,1\}^{n}$, and a function on the hypercube extends to a polynomial function if and only if it is monotone.

Let us now consider a distributive lattice $L$ without least and greatest elements. (We omit the analogous discussion of the cases where $L$ has one boundary element.) Polynomial functions over $L$ can still be given in DNF of the form 2.1 by allowing the coefficients $c_{I}$ to take also the values 0 and 1 , which are considered as external 
boundary elements (see, e.g., 1, 3]). For example, a polynomial function $p(x, y)=$ $a \vee x \vee(b \wedge x \wedge y)$ can be rewritten as $p(x, y)=a \vee(1 \wedge x) \vee(0 \wedge y) \vee(b \wedge x \wedge y)$.

We can still assume monotonicity of the coefficients, and any such system $c_{I} \in$ $L \dot{\cup}\{0,1\}(I \subseteq[n])$ of coefficients gives rise to a polynomial function $p$ over $L$, provided that $c_{\emptyset} \neq 1$ and $c_{[n]} \neq 0$. (The cases $c_{\emptyset}=1$ and $c_{[n]}=0$ correspond to the constant 1 and constant 0 functions.) Again, such DNF's will be called maximal disjunctive normal forms. Just like in the case of bounded lattices, there is a one-to-one correspondence between maximal DNF's and polynomial functions (cf. [1]). To see this, let us choose elements $a<b$ from $L$ to play the role of 0 and 1 , and let $\mathbf{e}_{I}$ be the "characteristic vector" of $I \subseteq[n]$ (i.e., the tuple $\mathbf{e}_{I} \in L^{n}$ whose $i$-th component is $b$ if $i \in I$ and $a$ if $i \notin I$ ). If $a$ is sufficiently small (less than all non-zero coefficients in the maximal DNF of $p$ ) and $b$ is sufficiently large (greater than all non-one coefficients in the maximal DNF of $p$ ), then a routine computation shows that

$$
p\left(\mathbf{e}_{I}\right)= \begin{cases}c_{I} & \text { if } c_{I} \in L, \\ a & \text { if } c_{I}=0, \\ b & \text { if } c_{I}=1\end{cases}
$$

This means that we can recover the coefficients $c_{I}$ of the maximal DNF of $p$ from certain values of $p$, namely we need to consider $p$ on larger and larger cubes $\{a, b\}^{n}$ by letting $a$ decrease and $b$ increase indefinitely. As the next example shows, this does not imply that there is only one polynomial function that takes prescribed values on a fixed cube $\{a, b\}^{n}$.

Example 2.3. Let $L$ be the lattice of open subsets of a topological space $X$, and let $a, b \in L$ with $a \subset b$. Since $L$ is a bounded distributive lattice, in view of (2.1), every unary polynomial function $p$ over $L$ can be represented by a unique maximal DNF of the form $p(x)=c_{0} \cup\left(c_{1} \cap x\right)$ with $c_{0}, c_{1} \in L, c_{0} \subseteq c_{1}$. It is straightforward to verify that such a polynomial function satisfies $p(a)=p(b)=b$ if and only if

$$
b \backslash a \subseteq c_{0} \subseteq b \text { and } b \subseteq c_{1} \subseteq X .
$$

Thus, there may be infinitely many polynomial functions $p$ whose restriction to the "one-dimensional cube" $\{a, b\}$ is constant $b$ (for instance, let $X$ be the real line, and let $a$ and $b$ be open intervals).

Let us go one step further, and choose a "zero" and "one", possibly different in each coordinate: Let $a_{i}, b_{i} \in L$ with $a_{i}<b_{i}$ for each $i \in[n]$, and let $\widehat{\mathbf{e}}_{I}$ be the "characteristic vector" of $I \subseteq[n]$ (i.e., the tuple $\widehat{\mathbf{e}}_{I} \in L^{n}$ whose $i$-th component is $b_{i}$ if $i \in I$ and $a_{i}$ if $i \notin I$ ). The task of finding a polynomial function (or rather all polynomial functions) that takes prescribed values on the tuples $\widehat{\mathbf{e}}_{I}$ can be regarded as an interpolation problem.

Problem 2.4. Let $L$ be a distributive lattice. Given $D:=\left\{\widehat{\mathbf{e}}_{I}: I \subseteq[n]\right\}$ and $f: D \rightarrow L$, find all polynomial functions $p: L^{n} \rightarrow L$ such that $\left.p\right|_{D}=f$.

Note that here $f$ is given on the vertices of a rectangular box (cuboid) instead of a cube as in Theorem 2.1. We will solve this problem in Section 3, thereby generalizing Goodstein's theorem. Let us note that the problem can be interesting also in the case of bounded lattices, for instance, if we do not have access to the values of the polynomial function on $\{0,1\}^{n}$, but only on some "internal" points. We will discuss such applications in Section 5 .

\section{MAin Results}

Throughout this section we assume that $L$ is a distributive lattice and that $D=$ $\left\{\widehat{\mathbf{e}}_{I}: I \subseteq[n]\right\}$ and $f: D \rightarrow L$ are given. Our goal is to find (the maximal DNF of) all $n$-ary polynomial functions $p$ over $L$ that satisfy $\left.p\right|_{D}=f$. Clearly, monotonicity of $f$ is a necessary condition for the existence of a solution of Problem 2.4. but, in contrast 
with Goodstein's theorem, monotonicity is not always sufficient in this more general setting. We will prove that the extra condition that we need is the following:

$$
f\left(\widehat{\mathbf{e}}_{I \cup\{k\}}\right) \wedge a_{k} \leq f\left(\widehat{\mathbf{e}}_{I}\right) \leq f\left(\widehat{\mathbf{e}}_{I \backslash\{k\}}\right) \vee b_{k} \quad \text { for all } I \subseteq[n], k \in[n] .
$$

Observe that the first inequality holds obviously if $k \in I$, and the second inequality is also clear if $k \notin I$.

Our first lemma shows how to obtain inequalities between $f\left(\widehat{\mathbf{e}}_{S}\right)$ and $f\left(\widehat{\mathbf{e}}_{T}\right)$ for $S \subseteq T$ by repeated applications of $\star \star$.

Lemma 3.1. If the function $f$ satisfies $(\star)$, then for all $S \subseteq T \subseteq[n]$ we have

$$
f\left(\widehat{\mathbf{e}}_{T}\right) \wedge \bigwedge_{k \in T \backslash S} a_{k} \leq f\left(\widehat{\mathbf{e}}_{S}\right) \text { and } f\left(\widehat{\mathbf{e}}_{T}\right) \leq f\left(\widehat{\mathbf{e}}_{S}\right) \vee \bigvee_{k \in T \backslash S} b_{k} .
$$

Proof. We only prove the first inequality; the second one follows similarly. Let $T \backslash$ $S=\left\{k_{1}, \ldots, k_{r}\right\}$, and let us apply (the first inequality of) condition ( $\star$ ) with $I=$ $S \cup\left\{k_{1}, \ldots, k_{m-1}\right\}$ and $k=k_{m}$ for $m=1, \ldots, r$ :

$$
\begin{aligned}
f\left(\widehat{\mathbf{e}}_{S \cup\left\{k_{1}\right\}}\right) \wedge a_{k_{1}} & \leq f\left(\widehat{\mathbf{e}}_{S}\right), \\
f\left(\widehat{\mathbf{e}}_{S \cup\left\{k_{1}, k_{2}\right\}}\right) \wedge a_{k_{2}} & \leq f\left(\widehat{\mathbf{e}}_{S \cup\left\{k_{1}\right\}}\right), \\
& \vdots \\
f\left(\widehat{\mathbf{e}}_{S \cup\left\{k_{1}, \ldots, k_{r}\right\}}\right) \wedge a_{k_{r}} & \leq f\left(\widehat{\mathbf{e}}_{S \cup\left\{k_{1}, \ldots, k_{r-1}\right\}}\right) .
\end{aligned}
$$

Combining these $r$ inequalities, we get

$$
f\left(\widehat{\mathbf{e}}_{S \cup\left\{k_{1}, \ldots, k_{r}\right\}}\right) \wedge a_{k_{1}} \wedge \cdots \wedge a_{k_{r}} \leq f\left(\widehat{\mathbf{e}}_{S}\right) .
$$

Let us now show that $\star$ is a necessary condition for the existence of a solution of Problem 2.4

Lemma 3.2. If there is a polynomial function $p$ over $L$ such that $\left.p\right|_{D}=f$, then $f$ is monotone and satisfies (太).

Proof. Assume that $p$ is a polynomial function that extends $f$. Since $p$ is monotone, $f$ is also monotone. To show that ( $\star$ holds, let us fix $I \subseteq[n]$ and $k \in[n]$, and let us assume that $k \notin I$ (the case $k \in I$ can be dealt with similarly). Let $\left(\widehat{\mathbf{e}}_{I}\right)_{k}^{x} \in L^{n}$ denote the $n$-tuple obtained from $\widehat{\mathbf{e}}_{I}$ by replacing its $k$-th component by the variable $x$. We can define a unary polynomial function $u$ over $L$ by $u(x):=p\left(\left(\widehat{\mathbf{e}}_{I}\right)_{k}^{x}\right)$. Using this notation, $\star$ takes the form $u\left(b_{k}\right) \wedge a_{k} \leq u\left(a_{k}\right)$. The maximal DNF of $u$ is of the form $u(x)=c_{0} \vee\left(c_{1} \wedge x\right)$, where $c_{0}, c_{1} \in L \cup\{0,1\}$. Using distributivity and the fact that $a_{k}<b_{k}$, we can now easily prove the desired inequality:

$$
\begin{aligned}
u\left(b_{k}\right) \wedge a_{k}=\left(c _ { 0 } \vee \left(c_{1}\right.\right. & \left.\left.\wedge b_{k}\right)\right) \wedge a_{k}=\left(c_{0} \wedge a_{k}\right) \vee\left(c_{1} \wedge b_{k} \wedge a_{k}\right) \\
=\left(c_{0} \wedge a_{k}\right) \vee\left(c_{1} \wedge a_{k}\right) & \leq c_{0} \vee\left(c_{1} \wedge a_{k}\right)=u\left(a_{k}\right) .
\end{aligned}
$$

To find all polynomial functions $p$ satisfying $\left.p\right|_{D}=f$, we will make use of the the fact that every distributive lattice can be embedded into a Boolean algebra. Moreover, if $L$ is a distributive lattice, then there is a Boolean algebra $B(L)$ containing $L$ as a sublattice of the lattice reduct of $B(L)$ such that the boundary elements of $L$ (if they exist) coincide with the boundary elements of $B(L)$, and $L$ generates $B(L)$ (as a Boolean algebra). This Boolean algebra $B(L)$ is determined uniquely up to isomorphism (see [12]). Note that whenever we consider polynomial functions over $B(L)$ in the sequel, we will always mean lattice polynomial functions (not Boolean algebra polynomial functions).

The complement of an element $a \in B(L)$ is denoted by $a^{\prime}$. Given a function $f: D \rightarrow L$, we define the following two elements in $B(L)$ for each $I \subseteq[n]$ :

$$
c_{I}^{-}:=f\left(\widehat{\mathbf{e}}_{I}\right) \wedge \bigwedge_{i \notin I} a_{i}^{\prime}, \quad c_{I}^{+}:=f\left(\widehat{\mathbf{e}}_{I}\right) \vee \bigvee_{i \in I} b_{i}^{\prime} .
$$

Observe that $c_{I}^{-} \leq c_{I}^{+}$, and if $f$ is monotone, then $I \subseteq J$ implies $c_{I}^{-} \leq c_{J}^{-}$and $c_{I}^{+} \leq c_{J}^{+}$. Let $p^{-}$and $p^{+}$be the lattice polynomial functions over $B(L)$ that are represented by 
the (maximal) DNF's corresponding to these two systems of coefficients as in (2.1). We will see that $p^{-}$and $p^{+}$are the least and greatest lattice polynomial functions over $B(L)$ whose restriction to $D$ coincides with $f$ (whenever there exists such a polynomial function). Recall that although $B(L)$ is a Boolean algebra, we will only consider polynomial functions over the lattice reduct of $B(L)$; complements will appear only in coefficients of DNF's of these lattice polynomials.

Lemma 3.3. If $f$ is monotone and satisfies $\star \star$, then $p^{+}\left(\widehat{\mathbf{e}}_{J}\right) \leq f\left(\widehat{\mathbf{e}}_{J}\right)$ for all $J \subseteq[n]$. Proof. Let us fix $J \subseteq[n]$ and consider the value of $p^{+}$at $\widehat{\mathbf{e}}_{J}$ :

$$
p^{+}\left(\widehat{\mathbf{e}}_{J}\right)=\bigvee_{I \subseteq[n]}\left(c_{I}^{+} \wedge \bigwedge_{j \in I}\left(\widehat{\mathbf{e}}_{J}\right)_{j}\right)=\bigvee_{I \subseteq[n]}\left(c_{I}^{+} \wedge \bigwedge_{j \in I \backslash J} a_{j} \wedge \bigwedge_{j \in I \cap J} b_{j}\right) .
$$

It is sufficient to verify that each joinand is at most $f\left(\widehat{\mathbf{e}}_{J}\right)$. Taking into account the definition of $c_{I}^{+}$, this amounts to showing that

$$
\left(f\left(\widehat{\mathbf{e}}_{I}\right) \vee \bigvee_{i \in I} b_{i}^{\prime}\right) \wedge \bigwedge_{j \in I \backslash J} a_{j} \wedge \bigwedge_{j \in I \cap J} b_{j} \leq f\left(\widehat{\mathbf{e}}_{J}\right)
$$

holds for all $I \subseteq[n]$. Distributing meets over joins, the left hand side of $(3.2)$ becomes

$$
\left(f\left(\widehat{\mathbf{e}}_{I}\right) \wedge \bigwedge_{j \in I \backslash J} a_{j} \wedge \bigwedge_{j \in I \cap J} b_{j}\right) \vee \bigvee_{i \in I}\left(b_{i}^{\prime} \wedge \bigwedge_{j \in I \backslash J} a_{j} \wedge \bigwedge_{j \in I \cap J} b_{j}\right)
$$

Let us examine each joinand of this expression. For each $i \in I$, the joinand involving $b_{i}^{\prime}$ equals 0 , since

$$
b_{i}^{\prime} \wedge \bigwedge_{j \in I \backslash J} a_{j} \wedge \bigwedge_{j \in I \cap J} b_{j} \leq b_{i}^{\prime} \wedge \bigwedge_{j \in I \backslash J} b_{j} \wedge \bigwedge_{j \in I \cap J} b_{j}=b_{i}^{\prime} \wedge \bigwedge_{j \in I} b_{j} \leq b_{i}^{\prime} \wedge b_{i}=0 .
$$

The joinand of $(3.3)$ that involves $f\left(\widehat{\mathbf{e}}_{I}\right)$ can be estimated using $\sqrt{\star}$ and Lemma 3.1 (with $T=I$ and $S=I \cap J$ ):

$$
f\left(\widehat{\mathbf{e}}_{I}\right) \wedge \bigwedge_{j \in I \backslash J} a_{j} \wedge \bigwedge_{j \in I \cap J} b_{j} \leq f\left(\widehat{\mathbf{e}}_{I}\right) \wedge \bigwedge_{j \in I \backslash(I \cap J)} a_{j} \leq f\left(\widehat{\mathbf{e}}_{I \cap J}\right) .
$$

Since $f$ is monotone, we have $f\left(\widehat{\mathbf{e}}_{I \cap J}\right) \leq f\left(\widehat{\mathbf{e}}_{J}\right)$, and this proves 3.2 .

The following lemma is the dual of Lemma 3.3 , and it can be proved by using the minimal conjunctive normal form of $p^{-}$(cf. Remark 2.2).

Lemma 3.4. If $f$ is monotone and satisfies $(\star)$, then $p^{-}\left(\widehat{\mathbf{e}}_{J}\right) \geq f\left(\widehat{\mathbf{e}}_{J}\right)$ for all $J \subseteq[n]$.

The estimates obtained in the previous two lemmas allow us to find all solutions of our interpolation problem over $B(L)$, whenever a solution exists.

Theorem 3.5. Let $L$ be a distributive lattice and let $D=\left\{\widehat{\mathbf{e}}_{I}: I \subseteq[n]\right\}$ and $f: D \rightarrow L$ be given, as in Problem 2.4. Suppose that $f$ is monotone and satisfies ( $\star$ ), and let $c_{I}^{-}$and $c_{I}^{+}$be the coefficients computed from $f$ as in (3.1), and let $p^{-}$and $p^{+}$be lattice polynomial functions over $B(L)$ given by the DNF's corresponding to these coefficients. Furthermore, let $p$ be an n-ary lattice polynomial function over $B(L)$ given by a maximal DNF with coefficients $c_{I} \in B(L)(I \subseteq[n])$. Then the following three conditions are equivalent:

(i) $\left.p\right|_{D}=f$;

(ii) for all $I \subseteq[n]$ the inequalities $c_{I}^{-} \leq c_{I} \leq c_{I}^{+}$hold;

(iii) for all $\mathbf{x} \in L^{n}$ we have $p^{-}(\mathbf{x}) \leq p(\mathbf{x}) \leq p^{+}(\mathbf{x})$.

Proof. Implication (ii) $\Longrightarrow$ (iii) is trivial. To prove (ii) $\Longrightarrow$ (ii), assume that $\left.p\right|_{D}=f$, i.e., $p\left(\widehat{\mathbf{e}}_{J}\right)=f\left(\widehat{\mathbf{e}}_{J}\right)$ for all $J \subseteq[n]$. Then we can replace $f\left(\widehat{\mathbf{e}}_{J}\right)$ by $p\left(\widehat{\mathbf{e}}_{J}\right)$ in the definition of $c_{J}^{-}$, and we can compute its value by substituting $\widehat{\mathbf{e}}_{J}$ into the maximal DNF of $p$ :

$$
\begin{aligned}
c_{J}^{-} & =f\left(\widehat{\mathbf{e}}_{J}\right) \wedge \bigwedge_{j \notin J} a_{j}^{\prime}=p\left(\widehat{\mathbf{e}}_{J}\right) \wedge \bigwedge_{j \notin J} a_{j}^{\prime}=\left(\bigvee_{I \subseteq[n]}\left(c_{I} \wedge \bigwedge_{i \in I}\left(\widehat{\mathbf{e}}_{J}\right)_{i}\right)\right) \wedge \bigwedge_{j \notin J} a_{j}^{\prime} \\
& =\bigvee_{I \subseteq[n]}\left(c_{I} \wedge \bigwedge_{i \in I \backslash J} a_{i} \wedge \bigwedge_{i \in I \cap J} b_{i} \wedge \bigwedge_{j \notin J} a_{j}^{\prime}\right) .
\end{aligned}
$$


If there exists $i \in I \backslash J$, then $a_{i} \wedge a_{i}^{\prime}=0$ appears in the joinand corresponding to $I$, hence we can omit each of these terms from the join, and keep only those where $I \backslash J=\emptyset:$

$$
c_{J}^{-}=\bigvee_{I \subseteq J}\left(c_{I} \wedge \bigwedge_{i \in I \backslash J} a_{i} \wedge \bigwedge_{i \in I \cap J} b_{i} \wedge \bigwedge_{j \notin J} a_{j}^{\prime}\right) \leq \bigvee_{I \subseteq J} c_{I}=c_{J} .
$$

This proves $c_{J}^{-} \leq c_{J}$. The inequality $c_{J} \leq c_{J}^{+}$can be proved by a dual argument.

Finally, to prove (iii) $\Longrightarrow$ (ii), let us assume that $p^{-} \leq p \leq p^{+}$holds in the pointwise ordering of functions. Applying Lemma 3.3 and Lemma 3.4 we get the following chain of inequalities for every $I \subseteq[n]$ :

$$
f\left(\widehat{\mathbf{e}}_{I}\right) \leq p^{-}\left(\widehat{\mathbf{e}}_{I}\right) \leq p\left(\widehat{\mathbf{e}}_{I}\right) \leq p^{+}\left(\widehat{\mathbf{e}}_{I}\right) \leq f\left(\widehat{\mathbf{e}}_{I}\right) .
$$

This implies $p\left(\widehat{\mathbf{e}}_{I}\right)=f\left(\widehat{\mathbf{e}}_{I}\right)$ for all $I \subseteq[n]$, therefore we have $\left.p\right|_{D}=f$.

Note that in Lemma 3.2 we did not make use of the fact that $p$ is a polynomial function over $L$ : the proof works also for lattice polynomial functions over $B(L)$. This fact together with Theorem 3.5 shows that monotonicity and property $(\star)$ of $f$ are necessary and sufficient for the existence of a solution of our interpolation problem over $B(L)$ (regarded as a lattice). This observation leads us to the following result.

Theorem 3.6. Let $L$ be a distributive lattice and let $D=\left\{\widehat{\mathbf{e}}_{I}: I \subseteq[n]\right\}$ and $f: D \rightarrow L$ be given, as in Problem 2.4. Let $c_{I}^{-}$and $c_{I}^{+}$be the coefficients computed from $f$ as in (3.1), and let $p^{-}$and $p^{+}$be the lattice polynomial functions over $B(L)$ given by the DNF's corresponding to these coefficients. A polynomial function $p: L^{n} \rightarrow L$ with $\left.p\right|_{D}=f$ exists if and only if $f$ is monotone and satisfies ( $\star$ ). In this case a polynomial function $p$ over $L$ verifies $\left.p\right|_{D}=f$ if and only if $c_{I}^{-} \leq c_{I} \leq c_{I}^{+}$holds for coefficients $c_{I} \in L \cup\{0,1\}$ of the maximal DNF of $p$ for all $I \subseteq[n]$. In particular, $p$ can be chosen as the polynomial function $p_{0}$ given by the DNF corresponding to the coefficients $c_{I}=f\left(\widehat{\mathbf{e}}_{I}\right)$ :

$$
p_{0}(\mathbf{x})=\bigvee_{I \subseteq[n]}\left(f\left(\widehat{\mathbf{e}}_{I}\right) \wedge \bigwedge_{i \in I} x_{i}\right) .
$$

Proof. The necessity of the conditions has been established in Lemma 3.2. To prove the sufficiency, we just need to observe that if $f$ is monotone and satisfies $(\star)$, then the polynomial function $p_{0}$ is a solution of Problem 2.4 by Theorem 3.5, as $c_{I}^{-} \leq$ $f\left(\widehat{\mathbf{e}}_{I}\right) \leq c_{I}^{+}$follows immediately from the definition of $c_{I}^{-}$and $c_{I}^{+}$. Since $f\left(\widehat{\mathbf{e}}_{I}\right) \in L$ for all $I \subseteq[n]$, the polynomial function $p_{0}$ is actually a polynomial function over $L$. The description of the set of all solutions over $L$ also follows from Theorem 3.5 .

Let us note that if $L$ is bounded and $a_{i}=0, b_{i}=1$ for all $i \in[n]$, then Theorem 3.6 reduces to Goodstein's theorem. Indeed, in this case $\star \star$ holds trivially, hence a solution exists if and only if $f$ is monotone. Moreover, we have $c_{I}^{-}=c_{I}^{+}=f\left(\widehat{\mathbf{e}}_{I}\right)$, hence $p_{0}$ (which is the same as the polynomial function $p$ given in Theorem 2.1) is the only solution of Problem 2.4 .

\section{VARIATIONS}

We have seen that monotonicity and property ( $\star$ ) are necessary and sufficient to guarantee the existence of a solution of Problem 2.4. The following example shows that these two conditions are independent, hence neither of them can be dropped.

Example 4.1. Let $L$ be a distributive lattice, let $a, b, c \in L$ such that $a<b<c$, and let $D=\{a, b\}$. Then the function $f: D \rightarrow L$ defined by $f(a)=b, f(b)=a$ satisfies (太) but it is not monotone, while the function $g: D \rightarrow L$ defined by $g(a)=a, g(b)=c$ is monotone but it does not satisfy (太).

Considering lattice polynomial functions over $B(L)$, Problem 2.4 has a least and a greatest solution, namely $p^{-}$and $p^{+}$, whenever a solution exists (see Theorem 3.5). On the other hand, the instance of Problem 2.4 considered in Example 2.3 has no least solution over $L$ itself (since usually there is no least open set containing $b \backslash a$ ), and 
a dual example shows that in general there is no greatest solution over $L$. However, if $L$ is complete, then such extremal solutions exist over $L$. To describe these, let us introduce the following notation. For an arbitrary $b \in B(L)$, we define the elements $\operatorname{cl}(b)$ and $\operatorname{int}(b)$ of $L$ by

$$
\operatorname{cl}(b):=\bigwedge_{\substack{a \in L \\ a \geq b}} a \text { and } \operatorname{int}(b):=\bigvee_{\substack{a \in L \\ a \leq b}} a .
$$

Completeness of $L$ ensures that these (possibly infinite) meets and joins exist, and one can verify that $\mathrm{cl}$ is a closure operator on $B(L)$ (the closed elements being exactly the elements of $L$ ), while int is the dual closure operator on $B(L)$ (also called as "interior operator").

Theorem 4.2. Let $L$ be a complete distributive lattice; let $D=\left\{\widehat{\mathbf{e}}_{I}: I \subseteq[n]\right\}$ and $f: D \rightarrow L$ be given, as in Problem 2.4, and let us assume that $f$ is monotone and satisfies (太). Let $c_{I}^{-}$and $c_{I}^{+}$be the coefficients computed from $f$ as in (3.1), and let $p^{-}$ and $p^{+}$be the lattice polynomial functions over $B(L)$ given by the DNF's corresponding to these coefficients. Then a polynomial function $p$ over $L$ is a solution of Problem 2.4 if and only if

$$
\operatorname{cl}\left(c_{I}^{-}\right) \leq c_{I} \leq \operatorname{int}\left(c_{I}^{+}\right)
$$

holds for the coefficients $c_{I}$ of the maximal DNF of $p$, for all $I \subseteq[n]$.

Proof. Theorem 4.2 follows directly from Theorem 3.6 since, by the very definition of $\mathrm{cl}$ and int, we have that $c_{I}^{-} \leq c_{I} \leq c_{I}^{+}$holds for a given $c_{I} \in L$ if and only if $\operatorname{cl}\left(c_{I}^{-}\right) \leq c_{I} \leq \operatorname{int}\left(c_{I}^{+}\right)$.

Problem 2.4 was solved in [13] in the particular case of finite chains. That paper deals with Sugeno integrals (cf. Section 5 ) instead of lattice polynomials; here we reformulate the criterion for the existence of a solution ([13, Theorem 3]) in the language of lattice theory.

Theorem 4.3 ([13]). Let $L$ be a finite chain, and let $D$ be an arbitrary subset of $L^{n}$. A function $f: D \rightarrow L$ extends to a lattice polynomial function on $L$ if and only if

$$
\forall \mathbf{a}, \mathbf{b} \in D: f(\mathbf{a})<f(\mathbf{b}) \Longrightarrow \exists i \in[n]: a_{i} \leq f(\mathbf{a})<f(\mathbf{b}) \leq b_{i} .
$$

Let us explore the relationship between Theorem 4.3 and Theorem 3.6 Our condition $(\star)$ is defined only for sets $D$ of the form $D=\left\{\widehat{\mathbf{e}}_{I}: I \subseteq[n]\right\}$, whereas (4.1) can be interpreted for any set $D \subseteq L^{n}$ for any distributive lattice $L$. Hence it is natural to ask whether Theorem 4.3 remains valid for arbitrary distributive lattices. As the following example shows, if $L$ is not a chain, then it can be the case that (4.1) is neither sufficient nor necessary for the existence of a solution of Problem 2.4 not even for the special kind of sets $D$ that we considered in this paper.

Example 4.4. Let $L=\{0,1, a, b\}$ be the lattice shown on Figure 1. Let $n=1$ and $D=\{0, b\}$, and define $f: D \rightarrow L$ by $f(0)=b, f(b)=a$ and $g: D \rightarrow L$ by $g(0)=a$, $g(b)=1$. Then $f$ trivially satisfies $(4.1)$, but $f$ is not monotone, hence it is not the restriction of any polynomial function. On the other hand, $g$ does not satisfy (4.1), although it is the restriction of the polynomial function $p(x)=x \vee a$ to $D$.

Observe that if $L$ is a chain, then (4.1) implies that $f$ is monotone (of course, this follows from Theorem 4.3 , but it is also easy to verify directly). As we have seen in Example 4.4 , this is not true for arbitrary distributive lattices. Thus we may want to require that $f$ is a monotone function satisfying (4.1). We will prove below that if $D$ is of "rectangular" shape, then monotonicity of $f$ and condition (4.1) are sufficient to ensure that $f$ extends to a polynomial function (although 4.1) is not necessary, as we have seen in Example 4.4.

Proposition 4.5. Let $L$ be a distributive lattice and $D=\left\{\widehat{\mathbf{e}}_{I}: I \subseteq[n]\right\}$ as in Problem 2.4. If $f: D \rightarrow L$ is monotone and satisfies (4.1), then there exists a polynomial function $p$ over $L$ such that $\left.p\right|_{D}=f$. 


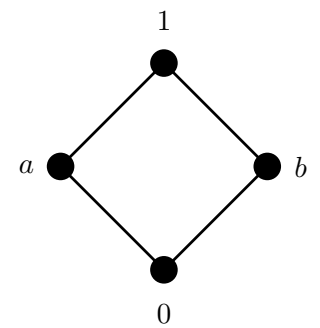

Figure 1. The lattice $L$ considered in Examples 4.4 and 4.6

Proof. Let $f: D \rightarrow L$ be a monotone function satisfying 4.1). By Theorem 3.6, we only have to prove that $f$ also satisfies $(\star)$. Let us assume that $k \notin I$; the other case is similar. Then only $f\left(\widehat{\mathbf{e}}_{I \cup\{k\}}\right) \wedge a_{k} \leq f\left(\widehat{\mathbf{e}}_{I}\right)$ needs to be verified, as the second inequality of $\star \star$ is trivial in this case. Since $f$ is monotone, we have $f\left(\widehat{\mathbf{e}}_{I}\right) \leq f\left(\widehat{\mathbf{e}}_{I \cup\{k\}}\right)$, and if equality holds here, then we are done. On the other hand, if $f\left(\widehat{\mathbf{e}}_{I}\right)<f\left(\widehat{\mathbf{e}}_{I \cup\{k\}}\right)$, then (4.1) implies that there is an $i \in[n]$ such that

$$
\left(\widehat{\mathbf{e}}_{I}\right)_{i} \leq f\left(\widehat{\mathbf{e}}_{I}\right)<f\left(\widehat{\mathbf{e}}_{I \cup\{k\}}\right) \leq\left(\widehat{\mathbf{e}}_{I \cup\{k\}}\right)_{i} .
$$

This is clearly impossible for $i \neq k$, since then the $i$-th component of $\widehat{\mathbf{e}}_{I}$ and $\widehat{\mathbf{e}}_{I \cup\{k\}}$ is the same. Thus we must have $i=k$, and then 4.2 reads as

$$
a_{k} \leq f\left(\widehat{\mathbf{e}}_{I}\right)<f\left(\widehat{\mathbf{e}}_{I \cup\{k\}}\right) \leq b_{k} .
$$

From this we immediately obtain the desired inequality:

$$
f\left(\widehat{\mathbf{e}}_{I \cup\{k\}}\right) \wedge a_{k} \leq a_{k} \leq f\left(\widehat{\mathbf{e}}_{I}\right) .
$$

Finally, we give an example that shows that monotonicity and condition (4.1) together do not guarantee the existence of a solution of Problem 2.4 if $L$ is an arbitrary distributive lattice and $D$ is an arbitrary subset of $L^{n}$. Thus it remains as a topic of further research to find an appropriate criterion for the existence of an interpolating lattice polynomial function in this general setting.

Example 4.6. Let $L$ be the same lattice as in Example 4.4 (see Figure 1), and let $D=\{a, b\}$. Then the function $f: D \rightarrow L$ defined by $f(a)=b, f(b)=a$ is monotone and satisfies (4.1), but it is not the restriction of a polynomial function. (This can be verified by a brute-force method, as there are only five nonconstant unary polynomial functions over $L$.)

\section{Application IN DECISION MAKING}

The original motivation for considering Problem 2.4 lies in the following mathematical model of multicriteria decision making. Let us assume that we have a set of alternatives from which we would like to choose the best one (e.g., a house to buy). Several properties of these alternatives could be important in making the decision (e.g., the size, price, etc., of a house), and this very fact can make the decision difficult (for instance, maybe it is not clear whether a cheap and small house is better than a big and expensive one). To overcome this difficulty, the values corresponding to the various properties of each alternative should be combined to a single value, which can then be easily compared.

To formalize this situation, let us assume that there are $n$ criteria along which the alternatives are evaluated, and these take their values in linearly ordered sets $L_{1}, \ldots, L_{n}$. These linearly ordered sets could be quantitative scales (e.g., $L_{1}$ could be the real interval $[40,200]$, measuring the size of a house in square meters) or qualitative scales (e.g., $L_{1}$ could be the finite chain $\{$ very small $<$ small $<$ big $<$ very big $\}$ ). Thus, to each alternative corresponds a profile $\mathbf{x} \in L_{1} \times \cdots \times L_{n}$. Since this product is usually not a linearly ordered set, some alternatives may be incomparable. Therefore, we choose a common scale $L$, and monotone functions $\varphi_{i}: L_{i} \rightarrow L(i \in[n])$ to translate 
the values corresponding to the different criteria (which may have different units of measure, e.g., square meters, euros, etc.) to this common scale, and which are then combined into a single value (for each alternative) by a so-called aggregation function $p: L^{n} \rightarrow L$. In this way we obtain a function $U: L_{1} \times \cdots \times L_{n} \rightarrow L$ defined by

$$
U(\mathbf{x})=p\left(\varphi_{1}\left(x_{1}\right), \ldots, \varphi_{n}\left(x_{n}\right)\right),
$$

and we can choose the alternative that maximizes $U$. The function $U$ is called a global utility function, whereas the maps $\varphi_{i}$ are called local utility functions. The relevance of such functions is attested by their several applications in decision making, in particular, in representing preference relations 2 .

It is common to choose the real interval $[0,1]$ for $L$, and consider $\varphi_{i}\left(x_{i}\right)$ as a kind of "score" with respect to the $i$-th criterion. In this case, simple aggregation functions $p$ are for instance the weighted arithmetic means, but there are of course other, more elaborate ways of aggregating the scores such as the so-called Choquet integrals. However, in the qualitative approach, where only the ordering between scores is taken into account (for instance, when $L=\{\operatorname{bad}<\mathrm{OK}<\operatorname{good}<$ excellent $\}$ ), such operators are of little use since they rely heavily on the arithmetic structure of the real unit interval. In the latter setting, one of the most prominent class of aggregation functions is that of discrete Sugeno integrals, which coincides with the class of idempotent lattice polynomial functions (see [1]).

In 5] and [6] a more general situation was considered: $L$ is an arbitrary finite distributive lattice, the lattice polynomial functions are not assumed to be idempotent, and the local utility functions are not assumed to be monotone (instead they have to satisfy the boundary conditions $\varphi_{i}\left(0_{i}\right) \leq \varphi_{i}\left(x_{i}\right) \leq \varphi_{i}\left(1_{i}\right)$ for all $x_{i} \in L_{i}$, where $0_{i}$ and $1_{i}$ denote the least and greatest element of $\left.L_{i}\right)$. The corresponding compositions (5.1) were called pseudo-polynomial functions, and several axiomatizations were given for this class of functions. Besides axiomatization, another noteworthy problem is the factorization of such functions: given a function $U: L_{1} \times \cdots \times L_{n} \rightarrow L$, find all factorizations of $U$ in the form (5.1). Such a factorization can be useful in real-life applications, when only the function $U$ is available (from empirical observations), and an analysis of the behavior of the local utility functions $\varphi_{i}$ and of the aggregation function $p$ could give valuable information about the decision maker's attitude.

Suppose that we have already found the local utility functions $\varphi_{i}$ (see [5] and 6] for a method to find them), and let $a_{i}=\varphi_{i}\left(0_{i}\right), b_{i}=\varphi_{i}\left(1_{i}\right)$. If $\mathbf{x} \in L_{1} \times \cdots \times L_{n}$ is such that $x_{i}=1_{i}$ if $i \in I$ and $x_{i}=0_{i}$ if $i \notin I$, then $U(\mathbf{x})=p\left(\widehat{\mathbf{e}}_{I}\right)$. Thus, if we know the global utility function $U$, then we have information about $\left.p\right|_{D}$, and we can use Theorem 3.6 to find all possible lattice polynomial functions $p$ that can appear in a factorization (5.1) of $U$. (Of course, one also has to take into account the other values of $U$, but this can be done by using the boundary conditions; see [6].)

The factorization procedure outlined above can be applied only if we have complete information about the global utility function $U$. However, in practical situations this is rarely the case: either $U$ is not defined everywhere (e.g., there do not exist houses with all possible combinations of sizes, prices, etc.) or we do not know all of its values (e.g., the decision maker did not express his/her preferences about all the alternatives). This fact gives rise to the following problem.

Problem 5.1. Let $L_{1}, \ldots, L_{n}$ be bounded posets and $L$ a distributive lattice. Given $D \subseteq L_{1} \times \cdots \times L_{n}$ and $f: D \rightarrow L$, find all pseudo-polynomial functions $p: L_{1} \times \cdots \times$ $L_{n} \rightarrow L$ such that $\left.p\right|_{D}=f$.

Considering polynomials instead of pseudo-polynomials we have the following analogous problem, which was already outlined in Section 1 .

Problem 5.2. Let $L$ be a distributive lattice. Given $D \subseteq L^{n}$ and $f: D \rightarrow L$, find all polynomial functions $p: L^{n} \rightarrow L$ such that $\left.p\right|_{D}=f$.

As mentioned in Section 4 . Problem 5.2 was solved in 13 in the case of finite chains, which is the most common setting in the qualitative theory of decision making. The 
general case is of interest not only to qualitative decision making, but it is also both natural and pertinent in the theory of distributive lattices. This constitutes a topic of current research being developed by the authors.

Acknowledgments. The authors would like to thank Gábor Czédli for helpful discussions as well as the referees and the editor for their valuable comments that helped improving the paper.

\section{REFERENCES}

[1] Behrisch, M., Couceiro, M., Kearnes, K., Lehtonen, E., Szendrei, Á.: Commuting polynomial operations of distributive lattices. Order 29, 245-269 (2012)

[2] Bouyssou, D., Dubois, D., Prade, H., Pirlot, M. (eds): Decision-Making Process- Concepts and Methods. Wiley-ISTE (2009)

[3] Couceiro, M., Lehtonen, E.: Self-commuting lattice polynomial functions on chains. Aequationes Math. 81, 263-278 (2011)

[4] Couceiro, M., Marichal, J.-L.: Characterizations of discrete Sugeno integrals as polynomial functions over distributive lattices. Fuzzy Sets and Systems 161, 694-707 (2010)

[5] Couceiro, M., Waldhauser, T.: Axiomatizations and factorizations of Sugeno utility functions. Internat. J. Uncertain. Fuzziness Knowledge-Based Systems 19, 635-658 (2011)

[6] Couceiro, M., Waldhauser, T.: Pseudo-polynomial functions over finite distributive lattices. In Liu, W. (ed.), ECSQARU 2011, LNCS (LNAI) vol. 6717, pp. 545-556. Springer, Heidelberg (2011).

[7] Davey, B. A., Priestley, H. A.: Introduction to Lattices and Order. Cambridge University Press, New York (2002)

[8] Goodstein, R. L.: The Solution of Equations in a Lattice. Proc. Roy. Soc. Edinburgh Sect. A 67, 231-242 (1965/1967)

[9] Grätzer, G.: Boolean functions on distributive lattices. Acta Math. Acad. Sci. Hungar. 15, 195-201 (1964)

[10] Grätzer, G.: General Lattice Theory. Birkhäuser, Berlin (2003)

[11] Marichal, J.-L.: Weighted lattice polynomials. Discrete Math. 309, 814-820 (2009)

[12] Peremans, W.: Embedding of a distributive lattice into a Boolean algebra. Indag. Math. 19, 73-81 (1957)

[13] Rico, A., Grabisch, M., Labreuche, Ch., Chateauneuf, A.: Preference modeling on totally ordered sets by the Sugeno integral. Discrete Appl. Math. 147, 113-124 (2005)

(M. Couceiro) Mathematics Research Unit, FSTC, University of Luxembourg, 6, Rue Coudenhove-Kalergi, L-1359 Luxembourg, Luxembourg

E-mail address: miguel.couceiro@uni.lu

(T. Waldhauser) Mathematics Research Unit, FSTC, University of Luxembourg, 6, RuE Coudenhove-Kalergi, L-1359 Luxembourg, Luxembourg, And, Bolyai Institute, University of Szeged, Aradi vértanúk tere 1, H-6720 Szeged, Hungary

E-mail address: twaldha@math.u-szeged.hu 